\title{
Cervical degenerative changes in idiopathic scoliosis patients who underwent long fusion to the sacrum as adults: incidence, severity, and evolution
}

\author{
Elisha Ofiram • Timothy A. Garvey • \\ James D. Schwender · Jill M. Wroblewski • \\ Robert B. Winter
}

Received: 6 June 2008/ Accepted: 17 November 2008/Published online: 3 February 2009

(C) Springer-Verlag 2009

\begin{abstract}
Background To date, there have been no published studies of the degenerative changes in the cervical spine in adult idiopathic scoliosis patients with thoracic and lumbar curves severe enough to require major reconstructive surgery.

Materials and methods The primary study group was 48 adult patients who had previously undergone a fusion from T10 or higher to the sacrum as an adult for idiopathic scoliosis. These were compared to 38 adults with unfused idiopathic scoliosis of $30^{\circ}-50^{\circ}$ and to 42 symptomatic adults presenting with cervical pain. Cervical degeneration was assessed using a new cervical degenerative index (CDI).

Results The amount of degenerative change seen in the cervical spine in the long-fusion group was significantly higher at baseline (just prior to the fusion) than the two control populations and became much higher at a mean follow-up of 8.5 years.

Conclusions This unique subgroup of patients, those having fusion from the thoracic spine to the sacrum as adults for adolescent idiopathic scoliosis, had a high incidence and severity of degenerative changes in their cervical spine. Due to the presence of advanced cervical degenerative changes prior to the fusion, it is not possible to blame the fusion as the main cause for these findings. These changes are either related to the thoracic and lumbar
\end{abstract}

E. Ofiram - T. A. Garvey · J. D. Schwender .

J. M. Wroblewski · R. B. Winter

The Twin Cities Spine Center, Minneapolis, MN, USA

E. Ofiram $(\bowtie)$

Chosen Specialties Clinics, Spine Clinic, 3 Shprinzak St., 64738 Tel Aviv, Israel

e-mail: eofiram@gmail.com deformities or are more likely due to this subgroup having a higher natural propensity for degenerative changes.

Keywords Idiopathic scoliosis ·

Degenerative cervical disease - Cervical spondylosis .

Long fusion to the sacrum

\section{Introduction}

The subject of degenerative changes in adjacent segments following spine fusion has been of considerable interest with a multitude of published studies in the lumbar fusion, cervical fusion, and deformity fusion literature [1, 2]. Since degenerative changes in the spine occur naturally in all of us, the question always arises as to whether the changes seen are caused by the fusion or are merely the expression of our natural history. To date, there is no study in the literature evaluating degenerative changes in the cervical spine following long fusion to the sacrum for symptomatic idiopathic scoliosis. We have chosen to study three patient populations: a group of 48 adults who had a spine fusion from the thoracic spine to the sacrum for scoliosis, a group of 38 adults with unfused idiopathic scoliosis of $30-50^{\circ}$, and a group of 42 adults presenting to our clinic with cervical complaints, but no scoliosis. These groups were compared using a new cervical degenerative index (CDI) [3].

\section{Materials and methods}

The study has been performed according to the Declaration of Helsinki and was approved by the Ethical Committee. Enrolled patients gave informed consent. The primary study group (group I) was 48 adults with adolescent idiopathic 
scoliosis who had a long fusion to the sacrum (from T10 or higher) as an adult. The average age at that surgery was 50 years (range 18-72 years). The fusion extended to the sacrum because of painful degenerative changes in the lower lumbar spine. The upper limit of the fusion was at T2 or T3 in $20(44 \%), \mathrm{T} 4$ or T5 in $16(36 \%)$, and T6-T10 in 14 $(20 \%)$. These patients were part of a previous study on the results and complications of such fusion surgeries [4]. All of these patients had had long postero-anterior and lateral radiographs, which included the cervical spine, both before and after their spine fusion surgery, and four-view short cervical radiographs at follow-up.

This group was compared to another study group (group II), 38 adult patients (mean age 35) with unfused thoracic idiopathic scoliosis of $30-50^{\circ}$. These patients also all had long postero-anterior and lateral radiographs.

A final group (group III) was composed of 42 randomly selected adult patients with symptomatic degenerative cervical spine problems. None of these had scoliosis or previous cervical spine surgery. Excluded were patients with congenital cervical anomalies, infections, tumors, ossification of the posterior longitudinal ligament (OPLL), diffuse idiopathic skeletal hyperostosis (DISH), or any inflammatory disease involving the cervical spine.

These three groups were not homogeneous as no attempt was made to have similar ages, proportion of genders, or symptoms. The method used to accurately quantify the radiographic degenerative cervical changes in all three groups was a new CDI developed at our center. It is a detailed quantitative analysis based upon Gore's qualitative criteria for cervical spondylosis, published in 1986 [5].

The CDI has been validated showing high intraobserver and interobserver reliability and reproducibility among experienced clinicians. The index assesses four factors: disc space narrowing, anterior and posterior osteophytes, end-plate and facet sclerosis, and anterior/posterior olisthesis. Each of these factors is rated on a four-point scale $(0,1,2,3)$ at five cervical levels: C2-C3, C3-C4, C4-C5, C5-C6, and C6-C7. Factor scores are then summed for the five levels giving a possible range from 0 (no degeneration at any level) to 15 (a maximum rating of three at all five levels) for any single factor. Table 1 shows the details of the factor ratings.

Table 1 Cervical degenerative index (CDI) factor scoring

\begin{tabular}{lllll}
\hline Factors & \multicolumn{4}{l}{ CDI score } \\
\cline { 2 - 5 } & 0 & 1 & 2 & 3 \\
\hline Disc space narrowing & None & $25 \%$ & $50 \%$ & $75 \%$ \\
Endplate or facet sclerosis & None & Minimal & Moderate & Severe \\
Osteophytes & None & $<2 \mathrm{~mm}$ & $2-4 \mathrm{~mm}$ & $>4 \mathrm{~mm}$ \\
Olisthesis & None & $<3 \mathrm{~mm}$ & $3-5 \mathrm{~mm}$ & $>5 \mathrm{~mm}$ \\
\hline
\end{tabular}

The CDI was calculated as the sum of all four factors at all five levels, giving a possible score ranging from 0 to 60 $(15 \times 4)$. The Student's $t$ test was then used to evaluate the differences between populations.

We also measured the Cobb angles of the thoracic and lumbar curves, the thoracic lordosis or kyphosis, the sagittal plumb line (global sagittal alignment) from the center of the $\mathrm{C} 7$ vertebral body relative to the anterior and superior corner of the sacrum, the coronal plumb line from the center of $\mathrm{C} 7$ relative to the mid-sacrum, and the $\mathrm{C} 2-\mathrm{C} 7$ translation, defined as the sagittal distance between the anterior wall of the $\mathrm{C} 2$ body and the anterior wall of the $\mathrm{C} 7$ vertebral body. These measurements were done to see if there were any correlations with the severity of the degenerative cervical changes.

\section{Results}

For the long-fusion group, significant degenerative scores were seen at all levels, ranging from 6.9 (SD 2.6) at C5-C6 to 3.1 (SD 1.8) preoperatively, and 8.8 (SD 2.6) at C5-C6 to 4.4 (SD 2.0) at $\mathrm{C} 2-\mathrm{C} 3$ at the 8-year follow-up (See Table 2).

Factor scores (disc space narrowing, sclerosis, osteophytes, olisthesis) were higher in the long-fusion group (group I) before surgery and even more elevated at followup as compared to either the nonfused adult scoliosis group (group II) or the symptomatic nonscoliotic group (group III).

For example, disc space narrowing was 5.7 preoperatively in group I, and 8.7 at follow-up, as compared to 1.6 for group II and 3.6 for group III. The details of this analysis can be seen in Table 3 for all four factors in all groups. All changes in group I from pre-operation to follow-up were statistically significant at $P<0.001$.

There was a positive correlation found between the CDI score and the magnitude of the thoracic scoliosis, the $\mathrm{C} 2-$ C7 translation, and the change in the thoracic kyphosis. There was no correlation found between the CDI score

Table 2 Comparing the summary of level scores of group I patients (those undergoing long-fusion surgery to the sacrum) before and after the fusion

\begin{tabular}{llllll}
\hline Levels: group I & \multicolumn{2}{l}{ Pre-fusion surgery } & & \multicolumn{2}{l}{ Post-fusion surgery } \\
\cline { 2 - 3 } & Mean & SD & & Mean & SD \\
\hline C2-C3 & 3.1 & 1.8 & & 4.4 & 2.0 \\
C3-C4 & 4.8 & 1.7 & & 6.7 & 2.5 \\
C4-C5 & 5.7 & 2.3 & & 7.8 & 2.4 \\
C5-C6 & 6.9 & 2.6 & & 8.8 & 2.6 \\
C6-C7 & 6.3 & 2.2 & & 8.2 & 1.9 \\
\hline
\end{tabular}


Table 3 Comparing the summary of factor scores in all groups

\begin{tabular}{|c|c|c|c|c|c|c|c|c|}
\hline \multirow[t]{2}{*}{ Factors } & \multicolumn{2}{|c|}{$\begin{array}{l}\text { AIS adult with } 30-50^{\circ} \\
\text { scoliosis, not fused }\end{array}$} & \multicolumn{2}{|c|}{$\begin{array}{l}\text { Long fusion to sacrum, } \\
\text { pre-fusion surgery }\end{array}$} & \multicolumn{2}{|c|}{$\begin{array}{l}\text { Long fusion to sacrum, } \\
\text { post- surgery }\end{array}$} & \multicolumn{2}{|c|}{$\begin{array}{l}\text { Symptomatic adult with cervical } \\
\text { degeneration }\end{array}$} \\
\hline & Mean & SD & Mean & SD & Mean & SD & Mean & SD \\
\hline Disc narrowing & 1.6 & 1.3 & 5.7 & 3.1 & 8.7 & 2.9 & 3.6 & 2.8 \\
\hline Sclerosis & 0.3 & 0.9 & 9.8 & 2.5 & 11.1 & 2.0 & 0.6 & 2.4 \\
\hline Osteophytes & 1.0 & 1.2 & 6.3 & 1.8 & 8.9 & 1.9 & 2.7 & 2.2 \\
\hline Olisthesis & 1.5 & 0.6 & 5.1 & 2.4 & 7.0 & 2.5 & 4.1 & 2.0 \\
\hline
\end{tabular}

Table 4 Variables correlated with the CDI score

\begin{tabular}{|c|c|c|c|c|c|c|}
\hline & \multicolumn{2}{|c|}{ CDI total change } & \multicolumn{2}{|c|}{ CDI total post-surgery } & \multicolumn{2}{|c|}{ CDI total pre-surgery } \\
\hline & Corr & $p$ val & Corr & $p$ val & Corr & $p$ val \\
\hline PA1cobbAng & 0.079 & 0.656 & -0.178 & 0.307 & -0.088 & 0.605 \\
\hline PA2cobbAng & 0.089 & 0.617 & 0.238 & 0.169 & -0.052 & 0.759 \\
\hline PA3cobbAng & 0.069 & 0.719 & $-0.390 *$ & $0.030^{*}$ & -0.201 & 0.262 \\
\hline CervLord & 0.010 & 0.957 & 0.062 & 0.721 & 0.213 & 0.206 \\
\hline ThorKy & $0.419^{*}$ & $0.012 *$ & -0.220 & 0.197 & $-0.445^{*}$ & $0.006^{*}$ \\
\hline LumLord & -0.321 & 0.060 & -0.014 & 0.934 & 0.314 & 0.058 \\
\hline C2-C7 Trans & 0.282 & 0.101 & -0.115 & 0.504 & $-0.331 *$ & $0.042 *$ \\
\hline C2plumbPS & 0.188 & 0.280 & 0.090 & 0.600 & -0.206 & 0.215 \\
\hline C7plumbPS & 0.102 & 0.558 & 0.113 & 0.511 & -0.127 & 0.447 \\
\hline C7plx & 0.012 & 0.943 & -0.121 & 0.483 & -0.174 & 0.295 \\
\hline
\end{tabular}

* Statistically significant correlations $(P<0.05)$

between the uppermost level of the fusion, the number of fusion levels, the cervical lordosis, the lumbar lordosis, the $\mathrm{C} 2$ sagittal plumbline, and the $\mathrm{C} 7$ coronal plumbline. The statistical details of these correlations are seen in Table 4 .

\section{Discussion}

Very little has been written on problems in the cervical spine in patients with adolescent idiopathic scoliosis. The first suggestion of a problem was in a 1980 paper by Moskowitz et al. [6] who reviewed 61 patients who had had a posterior spine fusion for some type of scoliosis 25 years previously (range 20-30 years). An unexpectedly high incidence of cervical complaints (57\%) was reported amongst those patients.

Edgar and Mehta [7] in their 1988 paper on long-term follow-up of fused and unfused idiopathic scoliosis patients found an incidence of cervicodorsal pain in $7.8 \%$ of the unfused patients and $17.6 \%$ of the fused patients.

In 1995, Hilibrand et al. [8] published a radiographic study of the sagittal alignment of the cervical spine in patients with adolescent idiopathic scoliosis. They noted an inverse correlation between the thoracic and the cervical alignment. The more lordotic the thoracic spine, the more kyphotic the cervical spine. As this was a study only of alignment and only in adolescents, no comments could be made regarding adult pain problems or assessment of cervical degeneration.

Although it might be tempting to state a "cause and effect" relationship between the scoliosis fusion surgery and the significant degenerative cervical changes seen at follow-up, our analysis shows that these changes were present prior to the fusion, and that these changes were more advanced than in either of the two control groups, neither of which had a fusion. This suggests that either the prefusion deformity or the natural history of adolescent idiopathic scoliosis predisposed the patients to degenerative cervical changes. We are not aware of any studies, other than our unfused adult idiopathic control group, that looked at the incidence or severity of degenerative cervical changes in adult patients with residuals of unfused adolescent idiopathic scoliosis.

There are, however, some studies relevant to the issue of degenerative changes in the lumbar spine in patients with adolescent idiopathic scoliosis. The most significant of those are the recently published studies from Sweden by Danielsson and Nachemson [9, 10]. They analyzed two groups of patients with adolescent idiopathic scoliosis, one fused and the other braced, and compared those with an age 
and sex-matched control group. Both treated groups had a mean follow-up after treatment of 22 years. Both treated groups had the same residual curvature, $37^{\circ}$. They had more lumbar pain, although mild, than the control group, but the pain level in the surgical group was slightly higher than in the braced group. Cervical pain was not analyzed. Was this increased pain in the two treated groups due to the residual lumbar curve, or was it due to a propensity for adolescent idiopathic patients to have more degeneration than nonidiopathic patients?

Since the reason for extending the fusion to the sacrum in our long-fusion group was always the presence of major degenerative changes in the lower lumbar spine, it is perhaps this specific group of adults with scoliosis and degeneration that also show degenerative changes in the cervical spine. Despite the multitude of other measurements done, few positive correlations were seen. There was a positive correlation between the magnitude of the thoracic scoliosis and the $\mathrm{CDI}$ score, the $\mathrm{C} 2-\mathrm{C} 7$ translation score, and the CDI, and a correlation between the sagittal change in the thoracic spine before and after surgery with the CDI. Surprisingly, the uppermost level of the fusion and the number of levels fused did not show significant correlation to the CDI.

As with most studies, this retrospective examination of the cervical spine in a highly specific subset of patients poses more questions than it answers. It has several deficits including the lack of an age and sex-matched control group of equally deformed adults with idiopathic scoliosis but no fusion, a matched control group of equally deformed adult patients with nonidiopathic scoliosis, and a correlation of the CDI with patient functional status.

This review of radiological incidence and severity of cervical degenerative changes revealed a significantly higher occurrence in adult idiopathic scoliosis patients scheduled to have spine fusion surgery from the thoracic spine to the sacrum as compared to two control populations.
At a mean follow-up of 8.5 years after surgery, the degenerative changes had significantly increased.

Conflict of interest statement The authors declare that they have no conflict of interest related to the publication of this manuscript.

\section{References}

1. Chen CS, Feng CK, Cheng CK, Tzeng MJ, Liu CL, Chen WJ (2005) Biomechanical analysis of the disc adjacent to posterolateral fusion with laminectomy in lumbar spine. J Spinal Disord Tech 18:58-65

2. Gheseli G, Wang JC, Bhatia NN, Hsu WK, Dawson EG (2004) Adjacent segment degeneration in the lumbar spine. J Bone Jt Surg Am 86-A:1497-1503

3. Ofiram E, Garvey TA, Schwender JD, Denis F, Perra JH, Transfeldt EE, Winter RB, Wroblewski JM (2009) Cervical degenerative index: a new quantitative radiographic scoring system for cervical spondylosis with interobserver and intraobserver reliability testing. J Orthop Traumat 10(1). doi: 10.1007/s10195-008-0041-3

4. Weistroffer JK, Perra JH, Lonstein JE, Schwender JD, Garvey TA, Transfeldt EE, Ogilvie JW, Denis F, Winter RB, Wroblewski JM (2008) Complications in long fusions to the sacrum for adult scoliosis: minimum five-year analysis of 50 patients. Spine 33(13): 1478-1483

5. Gore DR, Sepic SB, Gardner GM (1986) Roentgenographic findings of the cervical spine in asymptomatic people. J Orthopaed Traumatol 11:521-524

6. Moskowitz A, Moe JH, Winter RB, Binner H (1980) Long-term follow-up of scoliosis fusion. J Bone Jt Surg Am 62-A:364-376

7. Edgar MA, Mehta MH (1988) Long-term follow-up of fused and unfused idiopathic scoliosis. J Bone Jt Surg Br 70-B:712-716

8. Hilibrand AS, Tannenbaum DA, Graziano GP, Loder RT, Hensinger RN (1995) The sagittal alignment of the cervical spine in adolescent idiopathic scoliosis. J Pediatr Orthop 8:627-632

9. Danielsson AJ, Nachemson AL (2003) Back pain and function 22 years after brace treatment for adolescent idiopathic scoliosis: a case-control study-part I. Spine 28:2078-2085

10. Danielsson AJ, Nachemson AL (2003) Back pain and function 23 years after fusion for adolescent idiopathic scoliosis: a casecontrol study-part II. Spine 28:E373-E383 